\title{
THE PRE-OPERATIVE ASSESSMENT AND INVESTIGATION OF OPHTHALMIC PATIENTS
}

\author{
M. McKIBBIN \\ Bradford
}

\begin{abstract}
SUMMARY
This study was performed to document the prevalence of pre-existing medical disease amongst 105 patients awaiting ophthalmic surgery, and to assess the usefulness of investigations performed in accordance with the guidelines of the Joint Working Party on Anaesthesia in Ophthalmic Surgery. Patients attending the preoperative assessment clinic prior to ophthalmic surgery all had a full medical history taken and a clinical examination performed. Investigations were then ordered in accordance with the guidelines of the Joint Working Party on Anaesthesia in Ophthalmic Surgery. Pre-existing disease was present in $\mathbf{7 4}$ patients and abnormal results were obtained from 77 of the 318 investigations. Only 132 of the investigations were felt to be clinically indicated, and of the 77 abnormal results only 11 were unexpected. Despite the high prevalence of medical disease, patients awaiting ophthalmic surgery should be investigated only when clinically indicated and not on the basis of age or sex alone.
\end{abstract}

The pre-operative assessment is an important part of the overall management of patients undergoing ophthalmic surgery. It allows pre-existing medical disease, both known and unknown, to be recorded after a detailed clinical history and examination. Preoperative investigations may then be ordered so that the results are available before the date of admission. A decision as to the most suitable mode of anaesthesia can then be made in the light of the history, examination and investigations. This study shows the high frequency of pre-existing disease amongst ophthalmic patients and considers the indications for and benefits of pre-operative investigations.

\section{METHOD}

Over a 3 month period in 1994, 105 patients were seen in the pre-operative assessment clinic at Bradford Royal Infirmary. All patients had a full

From: Department of Ophthalmology, Royal Infirmary, Bradford BD9 6RJ, UK.

Correspondence to: Mr Martin McKibbin, Department of Ophthalmology, St James's University Hospital, Beckett Street, Leeds LS9 7TF, UK. medical history taken, followed by examination of the cardiovascular and respiratory systems and urinalysis. Other systems were examined as indicated. Laboratory investigations included a full blood count (FBC), blood glucose, urea and electrolytes, electrocardiogram (ECG) and a chest radiograph. Investigations were ordered in accordance with the recommendations of the Joint Working Party on Anaesthesia in Ophthalmic Surgery (Table I). ${ }^{1}$ These provide specific clinical indications for preoperative investigations and also recommend some investigations on the basis of age and sex alone, irrespective of the mode of anaesthesia.

Chest radiographs were ordered according to local hospital guidelines, which restricted the use of preoperative chest radiographs to patients with new symptoms or to those with chronic cardiorespiratory disease who had not had a chest radiograph performed in the preceding year. Other investigations were ordered if specifically indicated.

\section{RESULTS}

Of the 105 patients, 45 were male and 60 were female. The average age was 70.1 years with a range of 21-91 years. Only 11 patients were under 60 years of age. Cataract was the most common indication for surgery ( 78 patients), while 13 patients were awaiting trabeculectomy, 4 patients were awaiting combined

Table I. Recommendations of the Joint Working Party concerning pre-operative investigations

\footnotetext{
1. ECG: for patients over 60 years and those with symptoms or signs of cardiovascular disease, including ischaemic heart disease or hypertension

2. Chest radiograph: for patients with a history or signs of chronic lung disease or any suggestion of malignancy or pulmonary tuberculosis

3. Urea, creatinine and electrolytes: for patients over 60 years, those with renal disease, and those taking cardiac, renal or steroid drugs

4. Blood sugar estimation: for all diabetics and patients on steroids

5. Haemoglobin: for all women, men over 60 years, and those with signs of anaemia
} 
Table II. Significant pre-existing medical disease

\begin{tabular}{lc}
\hline Medical disease & No. of patients \\
\hline Angina & 22 \\
Myocardial infarction & 9 \\
Systemic hypertension & 21 \\
Cerebrovascular accident (incl. TIA) & 4 \\
Congestive cardiac failure & 4 \\
Valvular heart disease & 7 \\
Peripheral vascular disease & 1 \\
Atrial fibrillation & 4 \\
Diabetes mellitus & 13 \\
Obstructive airway disease & 27 \\
Anaemia & 3 \\
Malignant disease & 2 \\
Pulmonary tuberculosis & 1 \\
Addison's disease & 1 \\
\hline
\end{tabular}

TIA, transient ischaemic attack.

cataract and glaucoma surgery and 10 patients were due to have other procedures.

Following the clinical history and examination, significant pre-existing medical disease was noted in 74 of the 105 patients. The principal conditions are summarised in Table II. Ischaemic heart disease, including previous myocardial infarction, was the most common condition and was present in 31 patients. Obstructive airway disease was present in 27 , while 21 had systemic hypertension and 13 had diabetes mellitus. One or more cardiac, renal or steroid drugs were being taken by 43 patients.

A total of 318 investigations were ordered and the results were recorded in all cases. Only 132 of the investigations were felt to be clinically indicated and 186 were performed on the basis of age or sex alone. This is illustrated in Table III. Abnormal results were noted in 77 cases. Abnormal blood chemistry was detected in 28 of the investigations. Two patients had hypochromic microcytic anaemia with haemoglobin values of $10.3 \mathrm{~g} / \mathrm{dl}$ and $9.7 \mathrm{~g} / \mathrm{dl} ; 2$ patients taking diuretics had hyponatraemia with sodium levels of 126 and $130 \mathrm{mmol} / \mathrm{l} ; 14$ patients had raised urea levels with a range of $8.0-16.3 \mathrm{mmol} / \mathrm{l}$ and 9 patients had an elevated random blood glucose with a range of 7.5$21.0 \mathrm{mmol} / \mathrm{l}$, while 1 had a low blood glucose of 1.8 $\mathrm{mmol} / \mathrm{l}$. ECG abnormalities were found in the recordings from 39 patients. Of these, 14 had signs of ischaemia or infarction, 4 had signs of left ventricular hypertrophy, and 25 had arrhythmias including sinus

Table III. Details of all the investigations performed, those felt to be clinically indicated and those giving abnormal results

\begin{tabular}{lccc}
\hline Investigation & $\begin{array}{c}\text { No. } \\
\text { performed }\end{array}$ & $\begin{array}{c}\text { No. clinically } \\
\text { indicated }\end{array}$ & $\begin{array}{c}\text { Abnormal } \\
\text { results }\end{array}$ \\
\hline FBC & 98 & 5 & 2 \\
Urea and electrolytes & 98 & 53 & 16 \\
Glucose & 15 & 15 & 10 \\
ECG & 97 & 49 & 39 \\
Chest radiograph & 10 & 10 & 10 \\
\hline
\end{tabular}

Normal values: haemoglobin, 11-15 g/dl; sodium, 135-145 mmol/l; potassium, 3.5-5.0 mmol/l; bicarbonate, $23-31 \mathrm{mmol} / \mathrm{l}$; urea, 2.7-6.7 mmol/l; blood glucose, 3.5-6.6 mmol/l. bradycardia, sinus tachycardia, atrial fibrillation, bundle branch block and first or second degree heart block. Abnormal features were present on all 10 chest radiographs, consistent with the clinical diagnosis.

\section{DISCUSSION}

Pre-operative assessment is important for evaluating existing disease and for detecting unrecognised disease. In this sample, $70.5 \%$ of patients had significant pre-existing medical disease. In previous studies dealing with cataract patients exclusively, pre-existing disease was noted in $84 \%$ and $62.5 \%$ of patients. ${ }^{2,3}$ In this series, 31 patients had neither a history nor signs of pre-existing disease, with 20 of these aged 60 years or more. The average age of the patients in all groups was similar.

Abnormal results were found in $24.2 \%$ of the investigations, although $86 \%$ of these were felt to have been expected from the history and examination. ECG abnormalities were most common and were found in 39 of 97 investigations. A normal resting ECG was found in 12 patients with a history of ischaemic heart disease. Signs of ischaemia were observed in 4 patients, all aged over 70 years, without a history of angina or infarction. Arrhythmias were present in 25 recordings. Bundle branch block was noted in 2 patients without any relevant disease on history or examination. The remainder all had a history or signs of ischaemia, hypertension or valvular heart disease.

A total of 12 patients were diabetic, and 9 of these had an elevated random blood glucose while 1 had a low random glucose of $1.8 \mathrm{mmol} / \mathrm{l}$. Both patients with hyponatraemia were taking diuretics; neither patient required specific treatment for the hyponatraemia prior to surgery. None of the patients on diuretics were found to be hypokalaemic. The 2 anaemic patients were subsequently found to be iron deficient, and both had been pale on clinical examination. All 10 chest radiographs were performed on patients with a history of either cardiac failure or obstructive airway disease.

Despite the prevalence of pre-existing disease and the frequency of abnormal results, the need to postpone surgery or treat patients was uncommon. Only 1 patient had surgery postponed following the pre-operative assessment. This patient was found to have diabetes mellitus. Random and fasting blood glucose estimations were performed after the urinalysis tested positive for glucose; the random blood glucose was $21.0 \mathrm{mmol} / \mathrm{l}$. Other patients required new or altered treatment after surgery, with 2 having treatment for an iron deficiency anaemia while 2 others were started on treatment for hypertension. A further 4 patients, already known to be hypertensive, were found to be poorly controlled and required a treatment modification. 
Investigations were performed only when clinically indicated or in accordance with the Joint Working Party guidelines on age and sex. Of the investigations performed, only $41.5 \%$ were felt to be clinically indicated, and $58.5 \%$ were performed on the basis of age or sex alone and without any clinical indication (see Table III). Abnormal results were found in $24.2 \%$ of all investigations. When the investigations were clinically indicated, $50 \%$ of the results were abnormal, while only $5.9 \%$ of the investigations which were not specifically indicated were abnormal. Results in this latter group which were abnormal were considered to be unexpected. There were 11 unexpected results in 10 patients: 5 patients had mildly raised urea levels, 2 had right bundle branch block and 4 had silent ischaemia. None resulted in the planned mode of anaesthesia being changed, with 2 of the patients with unexpectedly raised urea levels, 1 patient with unexpected bundle branch block and 2 patients with silent ischaemia subsequently having surgery under general anaesthesia.

Pre-operative investigations should be performed only when the results are likely to influence management. None of the abnormal results, either expected or unexpected, influenced the choice as to the mode of anaesthesia. This decision is best taken at the preoperative assessment clinic, after the history and examination. It must take account of the patient's choice, the local circumstances and the experience of the surgeon. ${ }^{4}$ When this is done there is unlikely to be any change in the mode of anaesthesia chosen. Furthermore, only for those patients having surgery under general anaesthesia were the abnormal results considered to be of possible significance. The management of patients having surgery under local anaesthesia was not influenced.

Pre-operative investigations are expensive, especially when the number performed without a clinical indication is considered. In this hospital, current costs are $£ 2$ for an FBC, $£ 2.90$ for a urea and electrolyte estimation, $£ 2.50$ for a blood glucose, $£ 9$ for an ECG and $£ 20$ for a chest radiograph. ${ }^{5}$ In this series the majority of the abnormal results were to be expected from the clinical history and examination, and none had any direct influence on the management of patients having local anaesthetic procedures. Restricting investigations to cases in which they are specifically indicated, and with the mode of anaesthesia in mind, would make the pre-operative investigations more cost effective.

Despite the Joint Working Party recommendations, it is not always possible for an anaesthetist to supervise all ophthalmic procedures performed under local anaesthetic. Given the high prevalence of medical disease amongst ophthalmic patients, the possibility of peri-operative complications should always be considered, even when no pre-existing disease is present. Most of these complications are unexpected and cannot be predicted from the preoperative assessment and investigation, particularly those occurring after retrobulbar block. Should serious complications occur, the surgeon may become solely responsible for the patient. It is therefore necessary for ophthalmic surgeons operating on patients under local anaesthetic without an anaesthetist present to be familiar with the current techniques of resuscitation.

There is an increasing trend for most ophthalmic surgery to be performed with the patient under local anaesthetic, ${ }^{6}$ and $43 \%$ of patients in this series were having local anaesthetic procedures. Day-case surgery is increasingly being used for cataract patients. The pre-operative assessment is important in the decision as to which patients are suitable for day-case surgery, as well as in the choice of the most appropriate mode of anaesthesia. This is particularly important if the waiting time for surgery is long. Postoperative confusion can occur in elderly patients after both local and general anaesthetic, ${ }^{t}$ and this possibility should be borne in mind when listing patients for day-case surgery.

In conclusion, patients undergoing ophthalmic surgery often have other systemic diseases. Despite this, many pre-operative investigations are currently performed without any specific clinical indication. These inappropriate investigations rarely produce unexpectedly abnormal results which are significant and likely to influence the management of the patient. Choosing the most appropriate mode of anaesthesia in the pre-operative assessment clinic prevents the possibility of subsequent change. Patients should be investigated only when clinically indicated, and not on the basis of age and sex alone. This is particularly true for procedures performed with the patient under local anaesthesia.

I would like to express my thanks to Mr J. S. Hillman for his comments during the preparation of this manuscript.

Key words: Medical disease, Ophthalmic surgery, Pre-operative investigation.

\section{REFERENCES}

1. Royal College of Anaesthetists and Royal College of Ophthalmologists. Report of the Joint Working Party on Anaesthesia in Ophthalmic Surgery. March 1993.

2. Fisher SJ, Cunningham RD. The medical profile of cataract patients. Geriat Clin North Am 1985;1:339-44.

3. Gilvarry A, Eustace P. The medical profile of cataract patients. Trans Ophthalmol Soc UK 1982;102:502-4.

4. Rosen E. Anaesthesia for ophthalmic surgery. Br J Ophthalmol 1993;77:542-3.

5. Personal communication. Heads of Departments, Bradford Royal Infirmary, 1994.

6. Courtney P. The National Cataract Surgery Survey: I. Eye 1992;6:487-92. 